\title{
Challenges in neuro-otology
}

\section{Michael Strupp*}

Department of Neurology and IFB'MU, University of Munich, Campus Grosshadern, Munich, Germany

*Correspondence: michael.strupp@med.uni-muenchen.de

This article provides an overview of the current methods of diagnosis and treatment of patients with vertigo, dizziness, or imbalance, as well as an analysis of ongoing problems besetting the field of neuro-otology. Aims and challenges are also described with the hope of stimulating basic and clinical research on these very frequent symptoms.

Vertigo and dizziness are among the most frequent symptoms that patients experience at some time in their lives. With a lifetime prevalence of about $30 \%$ and an annual incidence that increases with age, vertigo and dizziness will become even more important in the future. Despite this high prevalence and burden of disease, considerable challenges remain.

- Most patients with acute vertigo and dizziness are uncertain who to consult, because their problem lies inbetween the specializations of ear, nose, and throat (ENT) and neurology, and in some cases, ophthalmology, internal medicine, or psychiatry.

- While neurologists are very familiar with the anatomy, physiology, and pathophysiology of the central nervous system (central vestibular and ocular motor systems), ENT physicians specialize in the peripheral vestibular and audiological systems. However, very few have detailed knowledge of both the central and peripheral systems. Moreover, most ENT physicians practice mainly as ENT surgeons, and therefore, they are not necessarily specialists on vertigo and dizziness.

- With a few exceptions (South Korea, and some centers in the USA, UK, and Australia) cooperation between neurology and ENT as well as neuro-otology and oto-neurology is far from perfect. The underlying diseases resulting in vertigo and dizziness cover a broad spectrum of anatomical structures. Possible etiologies include ischemia, infection, autoimmune disorders, mechanical inner ear disorders, psychosomatic, and somatoform disorders. Therefore, the approach to patients requires a broad-based knowledge of anatomy, physiology, pathophysiology, and pharmacology as well as in-depth practical experience with the different methods available for evaluating the vestibular and ocular motor systems.

- Medical education in neuro-otology is also less than optimal. The problem begins in Medical School, where the vestibular and ocular motor systems are assumed to be among the most complicated anatomical and physiological systems of humans. Therefore, most medical students are not attracted to this field, which may also explain their limited knowledge of the subject. This downward trend continues during post-graduate training for most neurologists and ENT physicians, and is further exacerbated by the above-mentioned overlapping of the two disciplines. Moreover, to establish a correct diagnosis, physicians must examine the vestibular, audiological, and ocular motor systems. Furthermore, many physicians have difficulties examining the ocular motor system and ultimately interpreting their findings. What is pathological and what do the findings mean? Thus, topographical anatomical diagnosis remains a challenge: it is imperative to be able to discriminate between peripheral and central vestibular lesions is imperative.

- The link between basic physiology and the modeling of the vestibular and the ocular motor systems, on the one hand, and their clinical implications and applications, on the other, are far from ideal. While we know the detailed workings of the vestibular and ocular motor systems and can model their function and dysfunction, this knowledge has not yet been fully integrated in clinical practice. Conversely, clinical findings have to be linked with basic knowledge in order to stimulate the knowledge of physiology and the modeling of these systems.
- State-of-the-art randomized controlled clinical trials on the frequent causes of vertigo and dizziness as well as their underlying disorders are still lacking. This is true for phobic postural vertigo, vestibular migraine, Menière's disease, vestibular paroxysmia, downbeat and upbeat nystagmus, episodic ataxia, and perilymphatic fistula.

- The number of studies available on the impact of vertigo and dizziness, as well as impaired vestibular function on patients' quality of life and functioning are limited.

- Long-term studies on the natural course of the different forms of vertigo, their recurrence rates, or the long-term efficacy of available treatments are nonexistent. This is true for most forms of vertigo and dizziness.

In brief our growing knowledge of the anatomy and physiology of the vestibular and related ocular motor systems, as well as basic and clinical research must still overcome a number of shortcomings and limitations to improve diagnosis, develop new diagnostic criteria, and better understand the pathophysiology of these disorders. Hopefully, new treatment strategies and state-of-the-art prospective clinical trials will improve the quality of life and functioning in patients with vertigo and dizziness.

\section{HOW CAN WE SOLVE THESE WORLDWIDE PROBLEMS?}

First, we must increase worldwide awareness of the impact of vertigo and dizziness. While acute vertigo and dizziness may be the first sign of severe neurological disorders such as brainstem or cerebellar ischemia, chronic vertigo, dizziness, and balance disorders - in particular, if patients are not aware of their cause and are not adequately treated - have a considerable socio-economic impact.

Second, it is very important to improve the cooperation between ENT, neurology, neuro-ophthalmology, ophthalmology, and psychiatry, because many patients fall 
between these different specialties. Such cooperation requires the establishment of specialized interdisciplinary centers, in which ENT specialists, neurologists, ophthalmologists, psychiatrists, psychologists, cardiologists, radiologists, and neuroradiologists closely collaborate and examine patients together as a team. An example of such a center is the newly established Integrated Center for Research and Treatment of Vertigo, Dizziness and Ocular Motor Disorders $\left(I F B^{L M U}\right)$ at the University of Munich. The German Ministry of Research and Education will fund this center with 5 million Euros per year for 5-10 years. This novel patient-centered approach will allow the establishment of new paradigms to overcome the boundaries between these disciplines and thus have an enduring effect.

Third, only such an interdisciplinary approach will increase the knowledge and improve the skills of medical students and even physicians experienced in this field. Interdisciplinary thinking will be imperative in addition to a specialized curriculum covering vertigo, dizziness, imbalance, ocular motor, and gait disorders. It would suffice to have just one or two such centers per country. This approach would also have a snowballing effect leading to the creation of special examinations and certificate of qualifications.

Fourth, diagnostic criteria for some of the vestibular and ocular motor disorders have to be better defined and then internationally recognized. The Bárány Society therefore recently proposed an initiative, led by Dr. Alexandre Bisdorff, to establish committees that would formulate such criteria for the different forms of vertigo and dizziness. This has implications for clinical practice as well as for further clinical trials.

Fifth, multicenter, multinational prospective randomized placebo-controlled trials are urgently needed for a number of these disorders. Such studies should also measure the quality of life and functioning whenever possible. Examples are:
- Menière's disease - there is still a plethora of recommended treatments for this disorder, including a salt-free diet, diuretics, aminoglycosides, corticosteroids, betahistine, change of pressure in the middle ear, and surgery.

- Vestibular neuritis - the potential efficacy of corticosteroids and physiotherapy must still be determined.

- Vestibular migraine - the acute effects of drugs (also used in the treatment of migraine with or without aura), as well as the prophylactic treatment with beta-blockers, topiramate, or valproic acid must be investigated.

- Perilymphatic fistula - the natural course of this disorder should be compared with canal plugging, canal resurfacing, as well as fixation of the stapes.

- Bilateral vestibulopathy - the efficacy of physiotherapy and possible efficacy of certain drugs to improve the gain of the vestibulo-ocular reflex must be evaluated.

- Vestibular paroxysmia - treatment with agents such as carbamazepine or oxcarbamazepine must be investigated.

- Episodic ataxia - the efficacy of acetazolamide (never established in a placebo-controlled trial) as well as the aminopyridines must be clarified.

- Phobic postural vertigo - the potential efficacy of cognitive behavioral therapy and pharmacotherapy, for example with selective serotonin-reuptake inhibitors, must be established.

- The mechanisms of upbeat and downbeat nystagmus, acquired pendular nystagmus, and other forms of nystagmus must be clarified.

- The anterior canal variant of benign paroxysmal positioning vertigo must also be elucidated, in order to evaluate diagnostic criteria, prevalence, and treatment. There is an ongoing debate on whether this variant exists at all.

Further, histopathological post-mortem studies will require the establishment of brain banks on frequent disorders like bilateral vestibulopathy, Menière's disease, downbeat nystagmus, and episodic ataxia, to further enhance our knowledge about the histological changes and to differentiate possibly different forms. Finally, vestibular prostheses are being developed for patients with a complete bilateral vestibular failure but they still have to be implemented in clinical use.

The long-term goals of these measures should be

1. to improve our knowledge of the pathological anatomy and pathophysiology of the different causes of vertigo and dizziness arising from the labyrinth, vestibular nerve, brainstem, and cerebellum. Based on this knowledge, new therapeutic paradigms can be developed, particularly to improve central vestibular compensation after peripheral and central vestibular lesions.

2. to define worldwide accepted diagnostic criteria for the different causes of vertigo and dizziness, which can also form the basis for future clinical trials.

3. to improve the quality of life and functioning of these patients with physiotherapy, pharmacotherapy, psychotherapy, or surgery after correct diagnoses have been made and specific therapies established.

Looking back at the developments in the field over the last 20 years, we are highly optimistic that most of these goals will be reached within the next 20 years.

Submission of manuscripts dealing with the above-mentioned aspects will be very welcomed at Frontiers in Neuro-otology.

Received: 10 August 2010; accepted: 10 August 2010; published online: 11 October 2010. Citation: Strupp M (2010). Challenges for neuro-otology. Front. Neur. 1:121. doi: 10.3389/fneur.2010.00121

This article was submitted to Frontiers in Neuro-otology, a specialty of Frontiers in Neurology.

Copyright (c) 2010 Strupp. This is an open-access article subject to an exclusive license agreement between the authors and the Frontiers Research Foundation, which permits unrestricted use, distribution, and reproduction in any medium, provided the original authors and source are credited. 